\title{
El trabajo con las familias ante las drogas porteras con un enfoque de derechos
}

\section{Working with families in the face of porter drugs with a rights approach}

\author{
Jannet Maribel Flórez-Ibarra \\ Universidad César Vallejo, Peru \\ ORCID: https://orcid.org/0000-0003-4166-6733 \\ Mitchell Alberto Alarcón Diaz \\ Universidad Nacional Mayor de San Marcos, Perú \\ ORCID: https://orcid.org/0000-0003-0027-5701 \\ July Rivera-Zamudio \\ Universidad Nacional Mayor de San Marcos, Perú \\ ORCID: https://orcid.org/0000-0003-1528-4360

\section{Karel Llopiz Guerra} \\ Universidad Central "Marta Abreu" de las Villas, Cuba \\ ORCID: https://orcid.org/0000-0002-1500-8000
}

Received 10-12-20 Revised 11-25-20

Accepted 02-13-21 On line 03-02-21

*Correspondence

Email: kllopiz@uclv.cu
Cite as:

Flórez-Ibarra, J., Alarcón, D., Rivera-Zamudio, J., \& Llopiz, K (2021). El trabajo con las familias ante las drogas porteras con un enfoque de derechos. Propósitos y Representaciones, 9(1), e1060. Doi: http://dx.doi.org/10.20511/pyr2021.v9n1.1060

(c) Universidad San Ignacio de Loyola, Vicerrectorado de Investigación, 2021. 


\title{
Resumen
}

Los niños y adolescentes se encuentran expuestos al nivel familiar, tienen una diversidad de factores de riesgo y entre ellas se encuentran las drogas porteras. El tabaquismo, desde un enfoque de derecho debe tenerse en cuenta para atenuar esta situación en el marco de la familia. Este trabajo tiene como propósito describir la influencia familiar ante el tabaquismo y las consecuencias que acarrea desde el marco epidemiológico, legal y social.

Palabras claves: Influencia familiar, legalidad, prevalencia de vida, tabaquismo.

\begin{abstract}
:
Children, adolescents and young people at the family level are exposed to a variety of risk factors such as street drugs. One of them, smoking, must be reconciled from a legal approach to mitigate this situation within the family. The purpose of this paper is to describe the family influence on smoking and its consequences from the epidemiological, legal and social point of view. Family history of smoking has a significant influence on tobacco consumption.
\end{abstract}

Keywords: Family influence, legality, lifetime prevalence, smoking.

\section{Introducción}

La Convención sobre los Derechos del Niño, en su artículo 29, plantea que los Estados partes convienen en que la educación del niño deberá estar encaminada a: "Desarrollar la personalidad, las aptitudes y la capacidad mental y física del niño hasta el máximo de sus posibilidades" UNICEF (2006)

Los padres son, ante las leyes del país, los responsables de la educación de sus hijos. La historia de las actividades educativas de la escuela con las familias y la comunidad es rica en enseñanzas (Castro 2017)

Entre los principales motivos del inicio del consumo de drogas legales se ubican: la curiosidad, la imitación a los padres, la presión del grupo de amigos, la aceptación social, la anticipación a la edad adulta y la rebeldía (Cruz, Llopiz, Molerio, 2019)

A nivel mundial, en los últimos años, han ocurrido cambios importantes en el tipo de sustancias predominantemente consumidas, con la progresiva estabilización del consumo de derivados cannábicos y el fenómeno de las "drogas de diseño", ligado a patrones de consumo en situaciones sociales determinadas (Rodríguez, Sit, Puentes, Rosales. 2016)

Entre los factores que influyen en la iniciación al tabaquismo se encuentra que es una conducta socialmente aprendida, con diferencias individuales, familiares, económicas, culturales y sociales; así como que constituye una curiosidad natural de imitación de la 
conducta de padres, hermanos, amigos y familiares, así como el deseo de aceptación social (Plá, Elizarde, Cárdenas, Solares, Nieves. 2016)

Exponerse al riesgo es característico de la etapa de la adolescencia, sobre todo a partir de la conducta de imitación que adquiere desde la familia, La misma se puede manifestar por las consecuencias que pueden traer estos factores de riesgo a la salud y porque influyen además en que afloren otros factores de riesgo que pueden expresarse como la entrada de otros.

Este artículo tiene como propósito describir la influencia familiar ante el tabaquismo como droga portera y las consecuencias que acarrea desde el marco epidemiológico, legal y social.

El adolescente está expuesto a factores de riesgo como el tabaquismo, en el cual la familia juega un papel muy importante. Aparece como un posible factor causal del surgimiento precoz del hábito de fumar la baja autoestima, y estar expuestos desde estadíos iniciales de la vida a modelos fumadores constituye el primer factor influyente, más aún si son valorados positivamente por el niño o adolescente. Esta situación se agudiza sobre todo si los adultos no solo consienten, sino que estimulan la adicción, aunque sea indirectamente: padres y madres que fuman, héroes y heroínas de los medios de comunicación que lo hacen (cine, televisión, etc.) (Cedeño, 2015)

Entre los factores que influyen en la iniciación al tabaquismo se encuentra que es una conducta socialmente aprendida, con diferencias individuales, familiares, económicas, culturales y sociales; así como que constituye una curiosidad natural de imitación de la conducta de padres, hermanos, amigos y familiares, así como el deseo de aceptación social. Plá, Et-al. (2016)

Entre muchos otros hábitos, se ha demostrado que el tabaquismo de los padres influye en los hijos. Geneva (2015), la comunicación y cohesión familiar es altamente influyente en el inicio y uso continuado del tabaco. Geneva (2014).

El secreto familiar es un patrón interactivo conformado por al menos dos miembros de su familia, usualmente la madre y un hermano(a), con la finalidad de mantener encubierto al adolescente fumador. Pérez, Mendieta (2017)

En Latinoamerica existen leyes que prohíben en el orden legal fumar en Centros hospitalarios, escuelas y centros de trabajos públicos, así como restaurantes, bares, cines y teatros.

Se ido adquiriendo cierta cultura en este tema pero aun así, se ha constituido un cuerpo jurídico legal que acoge las diversas gestiones que se conciben y hacen desde diferentes escenarios, todo ello sustentado en las políticas destinadas a la familia y a los niños y las niñas.

En Cuba, varias leyes y resoluciones como la Constitución de la República, el Código de la Niñez y la Juventud, el Código de Familia, el Código Penal, entre otros, aseguran los 
derechos de la niñez y la juventud cubana así como de la familia. Estos documentos legales surgen a partir de las diversas transformaciones sociales; teniendo siempre en cuenta la base jurídica desde la Declaración sobre los Derechos del Niño celebrada por la ONU en 1991.

Al respecto, resulta interesante que existen suficientes medidas de prohibición y de regulación establecidas, aunque no se han encontrado relacionados a la prohibición de la publicidad y patrocinio (Suarez, Galceran, Serrat, 2018)

Según la OMS (2015) la encuesta de representatividad nacional, estimó en el año 2010 que fumaban $23,7 \%$ de la población de 15 años y más. Fuma el $16 \%$ de las mujeres y $31 \%$ de los hombres. El 74,8 \%, se inició antes de los 20 años de edad; uno de cada 10 fumadores antes de los 12 años de edad y cuatro de cada diez entre 12 y 16. Mientras en la exposición al humo de tabaco, ajeno al hogar, se encontró que globalmente $41 \%$ de los adultos cubanos declaró vivir en hogares en los que se fuma.

De las anteriores consideraciones, estudios sociales y jurídicos apuntan que los antecedentes familiares de tabaquismo influyen de forma directa en el inicio del consumo de tabaco de los niños y adolescentes. Estos antecedentes, como factor de riesgo importante para el consumo de tabaco en los niños y adolescentes, aparecen tanto en publicaciones científicas, de ocio, así como en los medios de difusión masiva, a partir de que la familia se considera como la primera escuela, donde se educa y forma al sujeto, basado en un modelo para el desarrollo de su vida integralmente. Por lo que los resultados de este trabajo permiten describir la situación actual ante este flagelo y cómo se manifiesta la dinámica familiar.

La prevalencia mayor de iniciación tabáquica se encuentra generalmente en adolescentes del sexo masculino, donde la familia fuma. La influencia de determinados miembros de la familia se describe en la literatura como otro factor de riesgo importante.

Debe destacarse también como se muestra en el estudio, el enorme riesgo que tienen por el poder del ejemplo y la imitación de adquirir ese hábito los adolescentes cuya familia también lo hace. Aunque no predominan numéricamente en la casuística son importantes las consecuencias funestas que trae el hábito tabáquico en las adolescentes del sexo femenino, por su capacidad de embarazarse, su efecto negativo párala lactancia materna y someter al bebe al consumo pasivo (Cuéllar, Pérez, Quintana, Castro, 2017).

Otro elemento a considerar en la prevención del tabaquismo es la familia, pues la transmisión de información sobre hábitos de vida sana en el medio familiar y su congruencia con los comportamientos del adulto (predicar con el ejemplo) son las acciones más exitosas que pueden realizarse con los hijos; si, por el contrario, la familia los incita al mal hábito, fumando en su presencia y brindándoles el cigarrillo, les facilita la iniciación y el consumo de tabaco. Se considera la familia como la piedra angular de ayuda en la adolescencia por la influencia positiva que puede lograr en su comportamiento. Está bien esclarecido que muchos se inician en el consumo del tabaco, entre otros factores, por la observación repetida de la conducta del adulto. (OPS, 1999) 


\section{Conclusiones}

Las experiencias acumuladas en el estudio de las familias, la legalidad en materia de derechos y deberes y los riesgos a los cuales que pueden exponer a sus hijos al consumir tabaco, se convierte en prioridad de investigación y acción, basado en una influencia desde las políticas educativas, con un enfoque legal y de derecho social pàra estrechar relaciones entre los padres y educadores y de esta forma juntos conducir la formación integral de niños y adolescentes con una adecuada dinámica familiar.

\section{Referencias bibliográficas.}

Castro, P.L. Experiencias de la educación cubana en la atención a las familias de los escolares. Ponencia publicada en Memorias del Congreso Pedagogía 2017.

Cedeño Volkmar G. El tabaco, la cultura y la salud. Rev Costarric Salud Pública. Vol 24(1):43- Disponible en: https://www.scielo.sa.cr/pdf/rcsp/v24n1/art05v24n1.pdf

Cuéllar, Á. J.Pérez, I. M. Quintana, U. M. Castro, D, N. (2017). Caracterización del hábitotabáquico en adolescentes de un consultorio del médico de familia. Rev Medimay 2017 Ago;24(3). Disponible en: https://www.medigraphic.com/pdfs/revciemedhab/cmh-2017/cmh173d.pdf

Cruz, A. Llopiz, K, Molerio, O. (2019). Educación antitabáquica, percepción de riesgo e incidencia en el rendimiento académico de estudiantes universitarios de Ciencias Médicas Propós. represent. vol.8 no.1 Lima ene./abr. 2020. Disponible en: http://dx.doi.org/10.20511/pyr2020.v8n1.433

Garcia, R. R. et al. Influencia familiar en el tabaquismo de los adolescentes. Rev Cubana Hig Epidemiol [online]. 2008, vol.46, n.3. ISSN 1561-3003. Disponible en: http://scielo.sld.cu/scielo.php?script=sci_abstract\&pid=S1561 30032008000300009

Geneva. (2014). Global status report on alcohol and health. Geneva: WHO Disponible en: http://www.who.int/mediacentre/factsheets/fs345/en/

Pérez, H. E. Mendieta, I.G. (2017). Dimensión interaccional de las configuraciones familiares en adolescentes fumadores. Rev Cubana Salud Pública vol.43 no.4 Dsponible en: http://scielo.sld.cu/scielo.php?script=sci_arttext\&pid=S086434662017000400004

Plá, G. A. Et-al. Tabaquismo: valores e integralidad. Rev.Med.Electrón. vol.38 no.3 Matanzas mayo.-jun. 2016. Disponible en: http://scielo.sld.cu/scielo.php?script=sci_arttext\&pid=S168418242016000300017

Organización Mundial de la Salud. (2015). Tabaco Datos y Cifras. Nota descriptiva $\mathrm{N}^{\circ}$ 339 Julio de 2015. OMS. Ginebra [Internet]. 2015, [citado: 4 SEP 2017]; Disponible en: http://www.who.int/mediacentre/factsheets/fs339/es/ 
Organización Panamericana de la salud. Tabaco y alcohol en el marco familiar y escolar. Latinoamérica contra el cáncer. Washington DC: OPS; 1999.

Rev. Horiz. sanitario vol.17 no.3 Villahermosa sep./dic. 2018. https://doi.org/10.19136/hs.a17n3.2389

Rodríguez, Pérez, L. Sit, Pacheco. R. Puentes, Valle. D. Rosales, Domínguez. O. (2016). Consumo de drogas: reporte por tarjeta de enfermedad de declaración obligatoria. Municipio Plaza de la Revolución.Rev. Habanera de Ciencias Médicas. Volumen 16, Número $4 . \quad$ Disponible en: http://www.revhabanera.sld.cu/index.php/rhab/article/view/1744/1861

Plá, García. A. Elizarde, Gálvez. M. Cárdenas, Friera. E. Solares, Carreño. J. Nieves, Sardiñas. B. (2016). Tabaquismo: valores e integralidad. Rev.Med.Electrón. vol.38 no.3. Disponible en: http://scielo.sld.cu/scielo.php?script=sci_arttext\&pid=S168418242016000300017

Suárez, L. N. Galceran-Serrat. V. (2018). Normas jurídicas de prevención, control del tabaquismo y Convenio Marco para el Control del Tabaco en Cuba. Rev. Horiz. sanitario vol.17 no.3 Villahermosa Disponible en: http://www.scielo.org.mx/scielo.php?script=sci_arttext\&pid=S200774592018000300167

World No Tobacco Day 2015: Stop illicit trade of tobacco products. Geneva: WHO; 2005. Access: 2015/12/26. Available at: http://www.who.int/campaigns/no-tobaccoday/2015/event/en/

UNICEF (2006). La Convención sobre los Derechos del Niño. Disponible en: https://unesdoc.unesco.org/ark:/48223/pf0000231442_spa 